\title{
Hierarchical Approach to Identifying Fluid Flow Models in a Heterogeneous Porous Medium
}

\author{
Emil N. Musakaev ${ }^{1}$ (D), Sergey P. $\operatorname{Rodionov}^{1}$ and Nail G. Musakaev $^{1,2, *(D)}$ \\ 1 Tyumen Branch of the Khristianovich Institute of Theoretical and Applied Mechanics SB RAS, \\ 74 Taymyrskaya Str., 625026 Tyumen, Russia; musakaev91@gmail.com (E.N.M.); rodionovsp@bk.ru (S.P.R.) \\ 2 Department of Applied and Technical Physics, University of Tyumen, 6 Volodarskogo Str., \\ 625003 Tyumen, Russia \\ * Correspondence: musakaev68@yandex.ru; Tel.: +7-3452-682-745
}

Citation: Musakaev, E.N.;

Rodionov, S.P.; Musakaev, N.G.

Hierarchical Approach to Identifying Fluid Flow Models in a

Heterogeneous Porous Medium.

Mathematics 2021, 9, 3289. https://

doi.org/10.3390/math9243289

Academic Editor: Stefania Cherubini

Received: 15 November 2021

Accepted: 16 December 2021

Published: 17 December 2021

Publisher's Note: MDPI stays neutral with regard to jurisdictional claims in published maps and institutional affiliations.

Copyright: (C) 2021 by the authors. Licensee MDPI, Basel, Switzerland. This article is an open access article distributed under the terms and conditions of the Creative Commons Attribution (CC BY) license (https:// creativecommons.org/licenses/by/ $4.0 /)$.
Abstract: A three-dimensional numerical hydrodynamic model fairly accurately describes the processes of developing oil and gas fields, and has good predictive properties only if there are highquality input data and comprehensive information about the reservoir. However, under conditions of high uncertainty of the input data, measurement errors, significant time and resource costs for processing and analyzing large amounts of data, the use of such models may be unreasonable and can lead to ill-posed problems: either the uniqueness of the solution or its stability is violated. A well-known method for dealing with these problems is regularization or the method of adding some additional a priori information. In contrast to full-scale modeling, currently there is active development of reduced-physics models, which are used, first of all, in conditions when it is required to make an operational decision, and computational resources are limited. One of the most popular simplified models is the material balance model, which makes it possible to directly capture the relationship between reservoir pressure, flow rates and the integral reservoir characteristics. In this paper, it is proposed to consider a hierarchical approach when solving the problem of oil field waterflooding control using material balance models in successive approximations: first for the field as a whole, then for hydrodynamically connected blocks of the field, then for wells. When moving from one level of model detailing to the next, the modeling results from the previous levels of the hierarchy are used in the form of additional regularizing information, which ultimately makes it possible to correctly solve the history matching problem (identification of the filtration model) in conditions of incomplete input information.

Keywords: filtration; heterogeneous porous medium; mathematical model; material balance equation; identification; hierarchical approach

\section{Introduction}

Over the past decade, there has been a fairly steady upward trend in global oil consumption for the energy and chemical industries [1,2]. Petroleum companies conduct research to improve the methods and measures aimed at economically viable extraction of hydrocarbons from oil fields [3,4]. At the same time, an important phase in the development of oil reservoirs is the mathematical modeling of these fields.

The main goal of reservoir modeling is to build a model such that it allows one to obtain a reliable forecast of the reservoir's behavior, as well as to identify a general strategy for an optimal oil field development. With the development of reservoir simulation techniques-especially taking into account the evolutionary increase in computing speed and improvement in numerical methods [5-7] - the so-called traditional approach to modeling has improved or been made better [8,9]. The essence of which is to create a fine scale full field model (FFM) to provide better information about current and future reservoir performance, using a full physics simulator. Historically, this approach has a number of significant disadvantages [10]: high uncertainty of the input data, including lack 
of knowledge about the reservoir structure and properties; inconsistency of the models with the quality of input data and prior knowledge, focus on the complexity of models and unjustified rejection of simple engineering models and techniques; internal inconsistency of the models and their inconsistency at the level of interdisciplinary synthesis; time and resource costs for processing and analyzing a large amount of data in conditions when rapid decisions are required.

All these problems are growing when solving the main development problems associated with multivariate calculations, such as: the procedure for matching the filtration model to historical data, the problem of uncertainty quantification technique, the problem of waterflooding optimization. For example, in [11], when estimating uncertainties within the Bayesian methodology for a synthetic field, an ensemble of 81 realizations of "candidate models" is reproduced, where only four key uncertainties were identified. Obviously, a real field requires a more detailed analysis and more scenarios. Due to the extreme resource intensity of this task, in practice, such a detailed uncertainty quantification technique is rarely carried out, although, of course, this would increase the stability of the model and improve forecast accuracy.

Therefore, in contrast to FFM, reduced-physics models are being rapidly developed $[12,13]$. The reduced-physics models emerged as an attempt to overcome the problems previously mentioned-due to their flexibility in accommodating the required input data, irrespective of type and size-hence accelerating the decision-making workflow [14]. Thus, if only well data are known with high accuracy and reservoir parameters in the inter-well space are determined with a large error, it seems more appropriate to use, for example, models based on modern material balance equation solution or capacity resistance model (CRM) $[10,12,15,16]$, instead of a traditional approach with a full physics simulation. It all depends on the balance of the model complexity and the means that would be required to build it, as shown in [17] (Figure 1). These material balance models make it possible to directly establish a relationship between reservoir pressure, flow rates of injection and production wells, as well as the integral characteristics of the reservoir and the fluids saturating it (aquifer parameters, compressibility of fluids and rocks). The main advantage of CRM models and similar ones over FFM is the calculation speed, which allows to obtain a solution and estimate uncertainties within an hour, i.e., almost in real time by the standards of reservoir modeling [10].

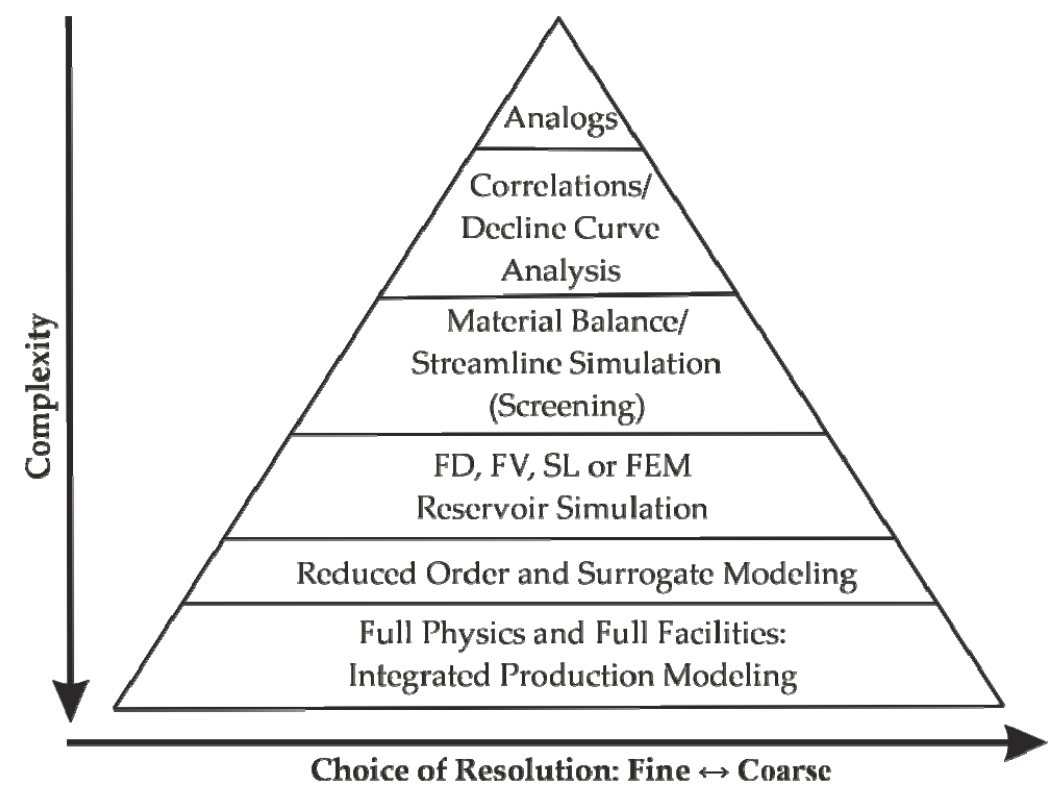

Figure 1. Classification of reservoir model reduction via complexity reduction [17].

Thus, an important task is to choose a model of optimal complexity that would describe the key features of the modeling object with an accuracy sufficient for practice. 
This is consistent with the principle of parsimony, which in the realities of mathematical modeling of complex objects can be formulated as follows: "the model should be as simple as possible and as complex as necessary" [14,18].

If we consider the problem of mathematical simulation of oil field development from the standpoint of a system approach, some principles of complex systems simulation should be followed, namely, the principle of model multiplicity and the principle of hierarchical modeling. As practice shows, following these principles is a time-consuming yet relevant task, especially for such resource-intensive development design steps as, for example, the reservoir system filtration model identification procedure. Therefore, to solve these problems, oil companies select highly qualified specialists with extensive modeling experience who are capable of systematizing heterogeneous, multi-scaled and inaccurate data into a single consistent FFM, actually derived from a variety of simple models and engineering techniques. At the same time, the development of new methods and algorithms within the framework of the hierarchical approach significantly reduces the load on a person, since many processes of transition from one level of the hierarchy to another are automated and do not require "manual" adjustments.

In this paper, it is proposed to consider a hierarchical approach in solving the problem of oil field waterflooding optimization using reduced-physics material balance models in successive approximations: first for the field as a whole (the field case), then for hydrodynamically connected blocks of the field (blocks case), then for the hydrodynamically connected wells (wells case). When moving from one level of model detail to the next, the modeling results from the previous levels of the hierarchy are used in the form of additional a priori information, which ultimately makes it possible to correctly solve the reservoir model identification problem in conditions of incomplete input data.

\section{Material Balance Model Hierarchy}

In the context of using reduced-physics models, there are widely spread models that describe waterflooding by number of hydrodynamically connected injection and production wells (Figure 2). These models include, for example, the capacity resistance model (CRM), the model based on the streamlines method (INSIM) (interwell numerical simulation model) [19,20], flow-network model [21], multiwell productivity index (MPI) -based method [22].

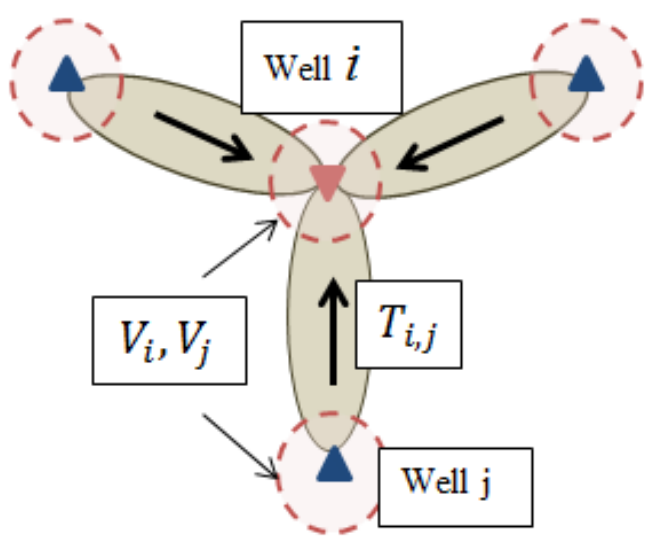

Figure 2. Model with a system of hydrodynamically connected injection and production wells.

In the general case of CRM models, a system of linear differential equations of material balance for liquid (water + oil) is solved, where the main input data are the volumes of injected $\left(Q_{i n j}\right)$ and produced fluids $\left(Q_{p r}\right)$, bottomhole pressures $\left(P_{w f}\right)$. The main matching parameters are the allocation factor $\left(f_{i, j}\right)$ between injection $(i)$ and production $(j)$ wells; the physical meaning of the relationship $f_{i, j}$ can be characterized as the ratio of the average 
transmissibility $\left(T_{i, j}\right)$ between injector and producer to the sum of the transmissibilities between the injection well and the associated production wells [10]:

$$
f_{i, j}=\frac{T_{i, j}}{\sum_{j=1}^{N_{p r}} T_{i, j}}
$$

where $N_{p r}$ is number of production wells.

In the INSIM model, in contrast to CRM, reservoir pressures are supplied across the wells, and the main matching parameters are transmissibilities between wells. In this case, not only are connections between production and injection wells evaluated, but also injection-injection, production-production connections.

In all the models under consideration, the number of matching parameters when solving the inverse problem is determined by the number of interwell connections $\left(N_{c}\right)$. In this case, the connectivity matrix, which initially establishes the presence and absence of connections between wells, must be determined in advance, based on some a priori information. If there are no restrictions on the connectivity matrix, then the number of adjustable parameters is equal to the maximum possible number of connections $N_{c, \text { max }}$, which is quadratic to the number of wells $\left(N_{w}\right)$ :

$$
N_{c, \max }=\frac{N_{w}}{2}\left(N_{w}-1\right)
$$

When solving an identification problem, this can lead to an ill-posed problem. Therefore, in practice, there are set restrictions on the interwell connections, based on the reservoir geometry and geology, restrictions on the length of the connection, etc. For example, in [22], a windowing method was proposed, where a windowing radius is set for each well. If adjacent wells in a circular area are determined by the windowing radius, then these wells are connected. For terrigenous reservoirs, without strong anisotropy of reservoir properties in space, this approach to determining the structure of the connectivity matrix is acceptable. However, for fractured-porous media, other methods of determining the matrix of connections are required, especially if there is no reliable data on the presence and location of highly permeable fractures and impermeable faults in the reservoir.

Material balance models in successive approximations. Let us consider the material balance equations $[9,23]$ for an oil field with a sequential complication of the model: the field as a whole or its isolated block, a system of hydrodynamically connected blocks or wells.

There is an oilfield divided into $N$ blocks; there are $N_{i n j}$ injectors and $N_{p r}$ producers; $N_{i n j}+N_{p r}=N_{w}$ (Figure 3). From the history of the field development, the injected volumes $Q_{i n j}^{k}(t)\left(k=1,2, \ldots, N_{i n j}\right)$ and production liquid rates $Q_{p r}^{k}(t)\left(k=1,2, \ldots, N_{p r}\right)$ are known. Average reservoir pressures around wells are also known $P_{\text {well }}^{k}(t)\left(k=1,2, \ldots, N_{w}\right)$. Then the reservoir pressure averaged over the $i$-th block $(i=1,2, \ldots, N)$, can be determined, for example, as the arithmetic mean reservoir pressure for all wells of the $i$-th block [24]:

$$
P_{i}=\frac{1}{N_{w, i}} \sum_{k=1}^{N_{w, i}} P_{w e l l}^{i}
$$

where $N_{w, i}$ is the number of wells at $i$-th block. 


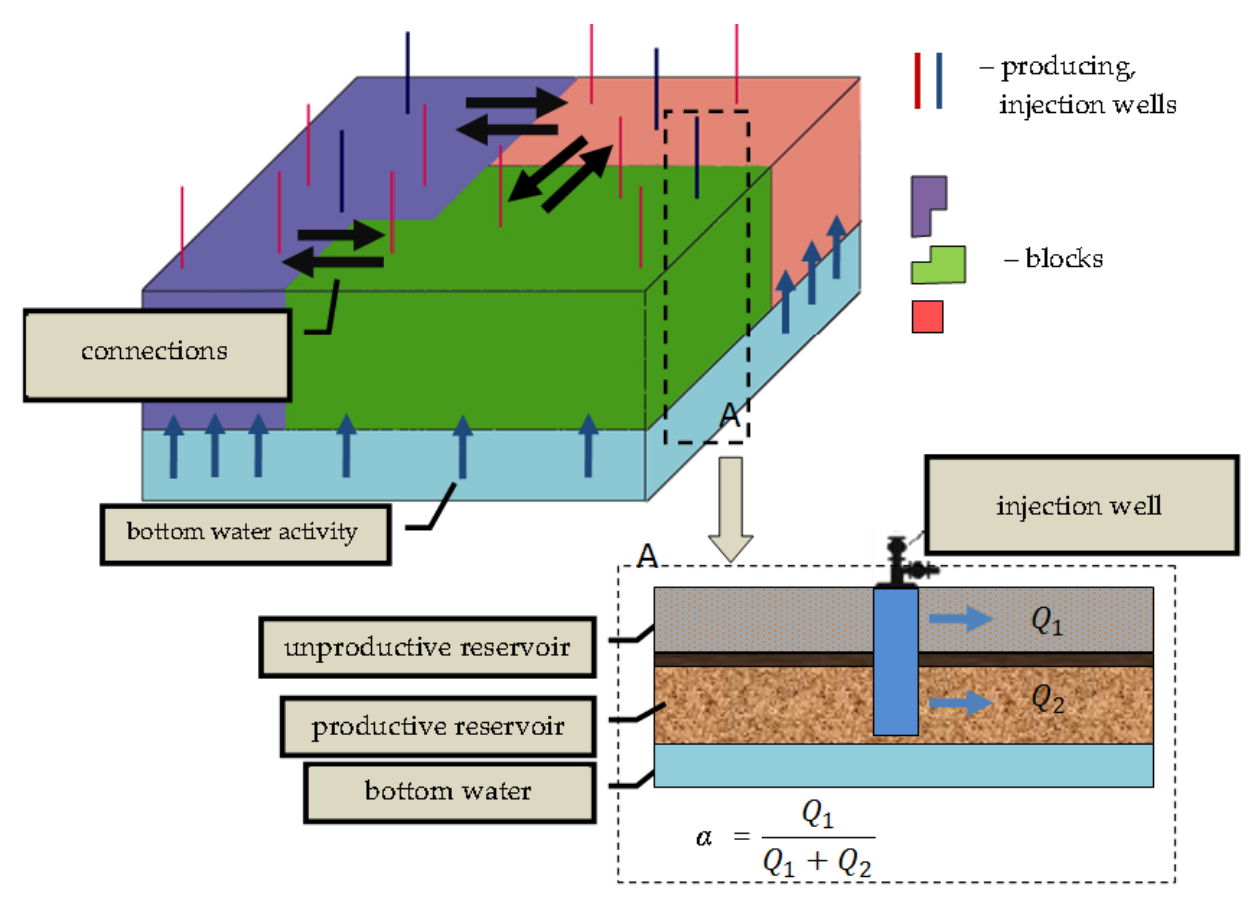

Figure 3. Schematic diagram of an oil field divided into blocks.

The field case. The object of modeling is the whole field, which is considered as one computational cell. The material balance equation for a homogeneous liquid can be represented as follows [24]:

$$
\beta V \frac{d P}{d t}=\alpha Q_{i n j}-Q_{p r}+\lambda_{a q}\left(P-P_{a q}\right)
$$

where $P$ is field average reservoir pressure; $P_{a q}$ is average bottom water pressure; $\beta$ is effective compressibility; $V$ is pore volume; $\alpha$ is effective injection ratio equal to the fraction of injected water that flows directly into the productive reservoir; $\lambda_{a g}$ is the coefficient, determining the intensity of bottom water inflow.

The vector of calculating parameters is written as follows:

$$
\vec{u}=\left(\beta, \alpha, \lambda_{a q}\right)
$$

Blocks case. The object of study is an oil field block, which is hydrodynamically connected with other blocks. Injected and produced fluid data are summed for each block, reservoir pressures are averaged over the block $P_{i}(2)$.

Let us write down the material balance equations for a system of $N_{b}$ hydrodynamically connected blocks [25]:

$$
\beta_{i} V_{i} \frac{d P_{i}}{d t}=\alpha_{i} Q_{i n j, i}-Q_{p r, i}-\lambda_{a q, i}\left(P_{i}-P_{a q, i}\right)+\sum_{j=1}^{n_{i}} \lambda_{i, j}\left(P_{i}-P_{j}\right)
$$

Here, the subscript $i\left(i=1,2, \ldots, N_{b}\right)$ marks the values related to the $i$-th block; $\lambda_{i, j}$ are coefficients characterizing the intensity of flows between blocks $i$ and $j$ (transmissibility between blocks); $n_{i}$ is the number of blocks connected with the $i$-th block.

Thus, in comparison with Equation (3), fluid flows between the blocks are added to the calculating parameters in the model with hydrodynamically connected blocks:

$$
\vec{u}=\left(\beta_{i}, \alpha_{i}, \lambda_{a q, i}, \lambda_{i, j}\right)
$$

If the coefficient $\lambda_{i, j}=0$, then it means that the blocks become isolated, and the system of Equation (5) is split into independent equations of the form (3). The dimension of the 
vector $\vec{u}$ depends on the number of connections between blocks $N_{c}$. At the maximum value, when each block is connected with others, the number of evaluating parameters is equal to:

$$
\operatorname{dim}(\vec{u})=3 N_{b}+\frac{1}{2} N_{b}\left(N_{b}-1\right)
$$

In general, the calculation of the material balance for a system of hydrodynamically connected blocks refers to the problem of sector modeling, when fluid flow between blocks is calculated and then serves as boundary conditions for a more detailed modeling of a separate area of an oil field [26].

Wells case. Let us represent the hydrodynamic model as a model with pairwise connections between wells $[16,27]$. The wells have "drainage volume" $V_{i}$. Flow in the interwell area between wells numbered $i$ and $j$ is modeled as flow within a region characterized by two parameters: reservoir transmissibility $T_{i, j}$ and a control pore volume $V_{p, i, j}$, where the transmissibility controls flow between wells in the interwell volume $V_{p, i, j}$. An algorithm for constructing a control volume between a pair of wells $V_{p, i, j}$ is shown in our work [18].

Let us determine the transmissibility between wells as follows [27]:

$$
T_{i, j}=\frac{B_{i j} K_{i j} H_{i j}}{L_{i j} \mu_{i j}}
$$

Here $K$ is the absolute permeability at the boundary between the wells; $B$ is the conditional width of the wall through which the liquid is filtered; $H$ is the arithmetic mean of the effective reservoir thickness; $L$ determines distance between wells; $\mu$ is the arithmetic average viscosity of the liquid. The liquid viscosity is determined through the initial water saturation $S_{m w}$ :

$$
\mu=S_{m w} \mu_{w}+\left(1-S_{m w}\right) \mu_{o}
$$

where $\mu_{w}$ and $\mu_{o}$ are water and oil viscosities.

The system of material balance equations for hydrodynamically connected wells is similar to the system of Equation (5) for the blocks:

$$
\beta_{i} V_{i} \frac{d P_{i}}{d t}=\alpha_{i} Q_{i n j, i}-Q_{p r, i}-\lambda_{a q, i}\left(P_{i}-P_{a q, i}\right)+\sum_{j=1}^{n_{i}} T_{i, j}\left(P_{i}-P_{j}\right)
$$

Here, the subscript $i$ marks the values related to the $i$-th well $\left(i=1,2, \ldots, N_{w}\right)$.

The vector of calculating parameters is expressed as follows:

$$
\vec{u}=\left(\beta_{i}, \alpha_{i}, \lambda_{a q, i}, T_{i, j}\right)
$$

where the maximum dimension of the vector $\vec{u}$, by analogy with (7), is equal to:

$$
\operatorname{dim}(\vec{u})=3 N_{w}+\frac{1}{2} N_{w}\left(N_{w}-1\right)
$$

If we take into account only the "production-injection" relationship, then the maximum dimension $\vec{u}$ is determined as follows:

$$
\operatorname{dim}(\vec{u})=3 N_{w}+N_{i n j} N_{p r}
$$

Representing systems of material balance equations in unified form. Material balance models for isolated blocks (3), for hydrodynamically connected blocks (5) or wells (9) are conveniently written in the following matrix form:

$$
\mathbf{V} \frac{d \mathbf{P}}{d t}=\mathbf{T P}+\mathbf{b}
$$


where $\mathbf{P}$ is a column vector with dimension $N$, which determines the reservoir pressure; $\mathbf{V}$ is an $N \times N$ diagonal matrix with elements $(V)_{i i}=\beta_{i} V_{i} ; \mathbf{b}$-column vector of dimension $N$ with elements $b_{i}=\alpha_{i} Q_{i n j, i}-Q_{p r, i}-\lambda_{a q, i} P_{a q, i} ; \mathbf{T}$-square $N \times N$ matrix of transmissibility coefficients with diagonal $(T)_{i, i}=\lambda_{a q, i}+\sum_{j=1}^{n_{i}} T_{i, j}\left(P_{i}-P_{j}\right)$ and off-diagonal elements $(T)_{i, j}=-T_{i, j}(i \neq j)$. For isolated sections, the matrix $\mathbf{T}$ is transformed into a diagonal matrix with elements $(T)_{i, i}=\lambda_{a q, i}$. The dimensionality of the matrices and vectors presented in (12) depends on the model under consideration: for blocks $N=N_{b}$, for wells $N=N_{w}$.

For the convenience of further presentation, we separate the connectivity matrix $\mathbf{A}$ (Figure 4) in the matrix of transmissibilities $\mathbf{T}$ through the element-wise product of matrices:

$$
\mathbf{T}=\mathbf{A} \circ \mathbf{T}
$$

where $\mathbf{A}$ is a symmetric binary matrix, which determines the absence of the hydrodynamic connection " $i-j$ " $(A)_{i, j}=0$ or its presence $(A)_{i, j}=1$. Thus, the connectivity matrix $\mathbf{A}$ determines the structure of the transmissibility matrix $\mathbf{T}$.

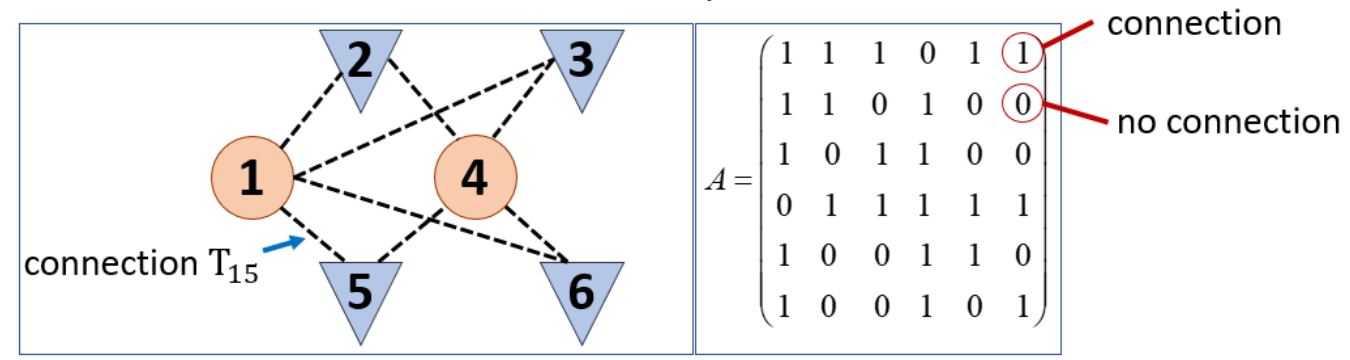

Figure 4. An example of setting the connectivity matrix $\mathbf{A}$ for injectors $\nabla$ and producers $\bigcirc$.

Material balance model identification. The solution to the inverse problem of identifying the model parameters will be the vector of calculating parameters $\vec{u}=\vec{u}_{\text {opt }}$, at which the objective function $J(\vec{u})$ has a global minimum $J\left(\vec{u}_{\text {opt }}\right) \rightarrow \min$. As an objective function, for example, a normalized measure of the deviation of the actual and calculated pressure values during the history period can be chosen:

$$
J(\vec{u})=\frac{\sum_{k=1}^{l}\left|P_{\text {fact }}^{k}-P_{\text {model }}^{k}(\vec{u})\right|}{\sum_{k=1}^{l} P_{\text {fact }}^{k}}
$$

where $l$ is number of time steps; $\vec{u}$ is vector of calculating parameters; $P_{\text {fact }}^{k}(k=1,2, \ldots, l)$ are reservoir pressure values calculated using production history data at time $t=t^{k}$; $P_{\text {model }}^{k}(\vec{u})(k=1,2, \ldots, l)$ are similar values of reservoir pressure, determined from the solution of Equation (9) using an evaluated vector of parameters $\vec{u}$.

As the model becomes more complex and the number of identifiable parameters increases, the inverse problem may turn out to be incorrectly posed. For instance, when identifying interwell transmissibility $\vec{u}=\left[T_{i, j}\right]$ in (9), the number of connections between wells $N_{c}$ (depending on the number of wells $N_{w}$ ) grows rapidly with a quadratic dependence (11), which, as practice shows, leads to non-uniqueness and instability of the solution. A forecast using such a numerical and/or mathematical model after parametric identification will be unstable or completely incorrect [14].

Therefore, it is necessary to involve additional regularizing information about the reservoir, the properties of the obtained solution, etc. In this work, it was proposed to consider the following properties of the obtained solutions:

1. Estimation of the predictive power of the model. For this, it is proposed to introduce a training data set $\vec{u}$ in which to identify parameters and test data in which to assess the 
quality of the model built. Thus, we divide the reservoir development period $T_{\text {all }}$ into the "training" period $T_{\text {training }}$ and the "test" period $T_{\text {test }}: T_{\text {all }}=T_{\text {training }}+T_{\text {test }}$. Parametric identification is performed on the intervals related to the "training" period, and the value of the functional will be obtained using Formula (14) $J_{\text {training }}\left(\vec{u}_{\text {opt }}\right)$. Knowing $\vec{u}=\vec{u}_{\text {opt }}$ and solving the direct problem during the "test" period, we obtain the value of the functional $J_{\text {test }}$. The general functional will then be equal to [18]:

$$
J_{\text {all }}=J_{\text {training }}+\gamma J_{\text {test }}
$$

where $\gamma$ is a weighting coefficient for the "test" period.

2. Taking into account the complexity of the selected model using the Bayesian information criterion $(B I C)$ in the following empirical form, analogous to the work [14]:

$$
B I C=\kappa \cdot \ln (n)+n \cdot \ln (J / n)
$$

where $J$ is the objective function (14) for the entire development period $T_{\text {all }} ; n$ is the number of observed values; $\kappa$ is parameter of the model complexity or the number of identifiable parameters. The model with the lowest BIC is preferred.

3. Taking into account a priori information $I_{a p r i o r i}$, obtained when simulating a complex real-world object with simplified reservoir models, which corresponds to a hierarchical approach to modeling.

Additionally, the work proposes a statistical approach to select the optimal model $\left(\boldsymbol{M}_{\text {opt }}\right)$ from a class of candidate models $\boldsymbol{M}_{\text {opt }} \in \boldsymbol{M}_{\text {class }}$. The idea of this approach is to statistically process the evaluation results after a series of calculations in candidate models, and use this information as criteria for selecting the optimal model.

Figure 5 shows a block diagram for selecting the optimal material balance model for the well case from the initially specified class of candidate models $\boldsymbol{M}_{\text {class }}$ using a priori information about reservoir data $\left(I_{\text {apriori }}\right)$. Each model differs from the other in the structure of the connectivity matrix (A). Having known the structure of the connectivity matrix, the transmissibility between the wells $(\mathbf{T})$ can be determined.



Figure 5. Block diagram of the methodology for structural-parametric identification of the material balance model. 
Statistical processing of the best $N_{m}$ models (best from the point of view $J_{s t r}$ ) can be carried out for each connection based on the frequency of the node occurrence in the $N_{m}$ best models:

$$
\left(p_{\text {stat }}\right)_{i, j}=\frac{1}{N_{m}} \sum_{n=1}^{N_{m}}(A)_{i, j}^{n}
$$

where $n=1,2, \ldots, N_{m}$ denotes the serial number of the model.

The following inequalities are used as criteria for selecting models:

$1.0 \leq\left(p_{\text {stat }}\right)_{i, j} \leq p_{\min }{ }^{\prime \prime} i-j "$ connection is absent;

2. $p_{\min } \leq\left(p_{\text {stat }}\right)_{i, j} \leq p_{\max }<<" i-j$ " connection is possible;

3. $p_{\max } \leq\left(p_{\text {stat }}\right)_{i, j}{ }^{\prime \prime} i-j$ " connection is defined.

The values $p_{\min }, p_{\max }$ are selected expertly. Thus, the set of criteria (17) allows for obtaining additional information $\left(I_{\text {stat }}\right)$ about the structure of the connectivity matrix and narrowing the class of candidate models $\boldsymbol{M}=\boldsymbol{M}\left(I_{\text {apriori }}, I_{\text {stat }}\right)$, from which the optimal model $\left(\boldsymbol{M}_{\text {opt }}\right)$ is selected.

One of the examples of the practical application of the hierarchical approach in identifying models of fluid flow in an inhomogeneous porous medium is presented in our work [28]. Another example is presented in Section 3.

\section{Application of the Hierarchical Approach in Calculating the Material Balance in the Field}

Let the oil field be expertly divided into $N=6$ blocks, for which the values of well flow rates and average reservoir pressures were defined throughout the development history. The schematic arrangement of the blocks is shown in Figure 6.



Figure 6. Schematic arrangement of blocks and representation of transmissibilities between them.

It is necessary to calculate the material balance for the system of hydrodynamically connected sections to determine the parameters $\vec{u}=\left(\alpha_{i}, \lambda_{a q, i}, \lambda_{i, j}\right)$ for each $i$-th block $(I=1,2, \ldots, N)$. According to (7), the number of identified parameters for the system 
under consideration is 27 for six equations. The set problem is incorrect; regularization is required.

Let us apply a hierarchical approach to calculating the material balance to regularize the problem. The hierarchical approach includes two stages that differ from each other in the form of the objective function and a set of control parameters [19]. In the first stage, we assume that there are no fluid flows $\lambda_{i, j}$ between the blocks, i.e., blocks of the field are isolated. In this case, the system of Equation (5) splits into independent Equation (3); the identifiable parameters are $\vec{u}=\left(\alpha_{i}, \lambda_{a q, i}\right)$. In the second stage, the vector of control parameters is formed by coefficients characterizing the intensity of fluid flows between the blocks $\vec{u}=\left[\lambda_{i, j}\right]$. The parameters $\alpha_{i}$ and $\lambda_{a g, i}$ for each block when solving the system of Equation (5) in the second stage are "frozen" and remain equal to the values obtained in the first stage.

Dividing the algorithm for solving the identification problem into two stages makes it possible to reduce the number of parameters identified simultaneously. As a result, the stability of the results obtained is increased, and the calculation time is also reduced.

When calculating the material balance for the isolated blocks, poor results were obtained for the identification of the parameters while minimizing the target function $J(\vec{u}) \rightarrow \min$. The problem lies in the non-uniqueness of the obtained optimal solution. Therefore, an additional regularization was carried out at the first stage of calculating the material balance using the tools of the theory of fuzzy sets. To construct the objective function, two fuzzy criteria were selected. In the first of them, the following expert statement is used for the parameter $\alpha$ (the subscript $i$ is omitted for simplicity): "the losses of the injected water should not be large". To do this, the following identity function was defined on the set of values of the coefficient $\alpha$, i.e., on the interval $0 \leq \alpha \leq 1$ :

$$
\varphi_{\alpha}=\varphi_{\alpha}(\alpha, 0,1,0.5)
$$

where

$$
\varphi(z, a, b, m)=\left\{\begin{array}{c}
1, z<m \\
\left(1-\frac{z-a}{b-a}\right)^{m}, a \leq z<b \\
0 . z \geq b
\end{array}\right.
$$

In this expression, $a$ and $b$ are numbers that are assigned by the expert as unconditionally small and unconditionally large, respectively; the parameter $m$ characterizes the degree of the decrease in confidence in the smallness of the number $z$ when it deviates from $a$. The membership function graph is shown in Figure 7a.

a

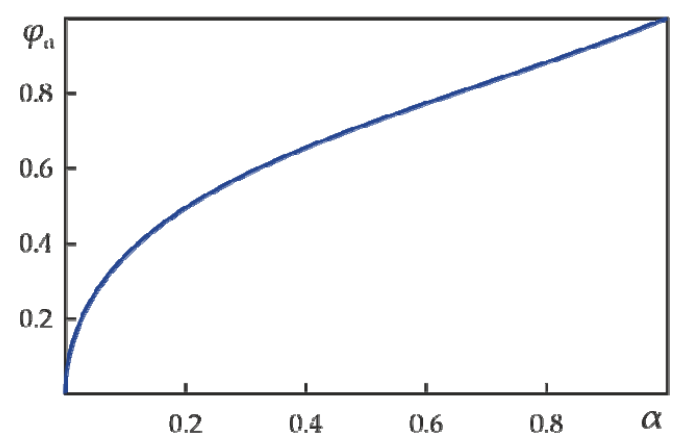

b

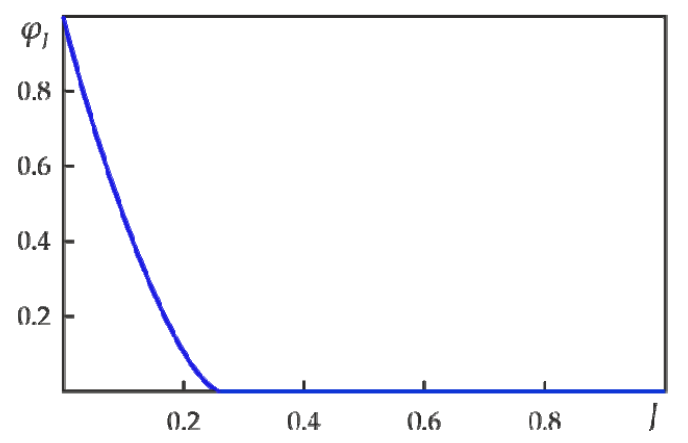

Figure 7. Membership functions (a) "injected water losses should not be large", (b) "the deviation of the calculated reservoir pressure from the actual one should not be large". 
The second fuzzy criterion was chosen in accordance with the following regularizing statement: "the deviation of the calculated and actual data should not be large". To formalize this criterion, the following membership function was chosen (Figure $7 \mathrm{~b}$ ):

$$
\varphi_{J}=\varphi_{J}(J, 0,0.3,2)
$$

where $J=J(\vec{u})=J\left(\alpha, \lambda_{a q}\right)$, a certain measure of deviation of calculated and actual data (8).

To combine these two criteria, the operation of intersection of fuzzy sets was used:

$$
J_{1}\left(\alpha, \lambda_{a q}\right)=\sqrt{\varphi_{\alpha} \varphi_{J}}
$$

As a result, the problem of identifying control parameters at the first stage is reduced to minimize the objective function $-J_{1}\left(\alpha, \lambda_{a q}\right)$. As an example, Figure 8 shows the objective function for one of the blocks before and after regularization. The function $J\left(\alpha, \lambda_{a q}\right)$, characterizing the deviation of the calculated pressure from the actual one does not have a pronounced minimum, which leads to the instability of the inverse problem solution. The function $-J_{1}\left(\alpha, \lambda_{a q}\right)$, on the other hand, has a single pronounced minimum. Thus, as a result of regularization based on the formalization of the expert assessment, the minimum of the function after the regularization becomes more pronounced, which makes it possible to obtain a more stable solution to the problem of identifying the control parameters included in the system of material balance equations.
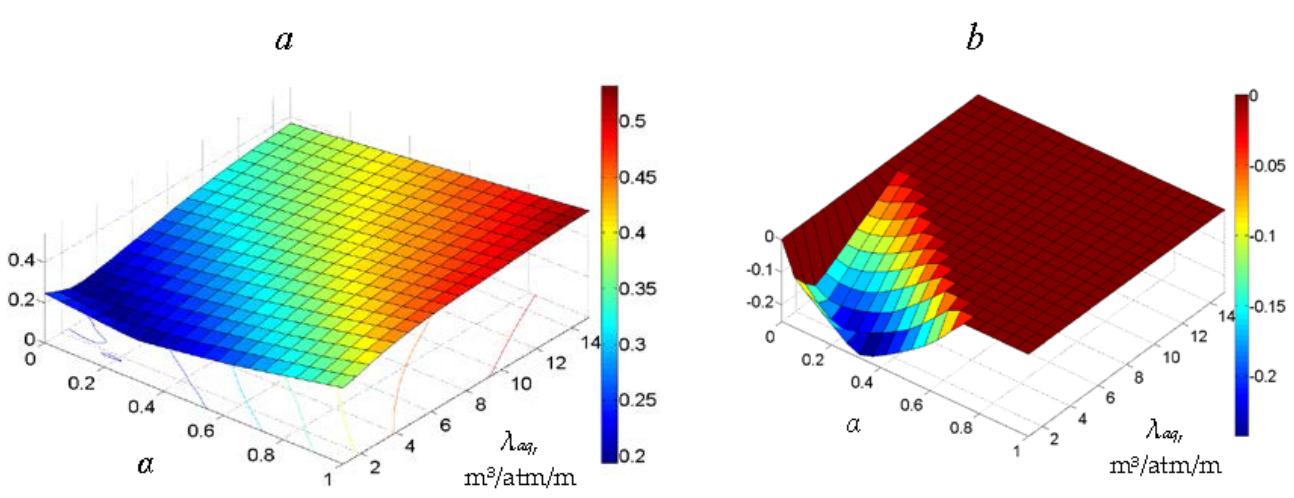

Figure 8. Comparison of the two types of objective functions $J\left(\alpha, \lambda_{a q}\right)(\mathbf{a})$ and $-J_{1}\left(\alpha, \lambda_{a q}\right)(\mathbf{b})$.

In the second stage of the algorithm, the inclusion of regularizing constraints in the objective function can be carried out by analogy to the first stage. For the formation of constraints, there is a priori information about the connections between the blocks, which can be investigated, for instance, from the results of hydrotesting of wells or tracer studies. In this case, those connections between blocks that geometrically intersect the perimeter of another block were not evaluated. For example, there is no connection between blocks " $2-4$ " as it crosses the perimeter of block " 3 " (Figure 6).

The initial values of the control parameters for the first stage of the algorithm are presented in Table 1. It also presents the results obtained after the parameters identification (Table 1 shows the values of the objective function $J$, but the function $J_{1}$ was used for the calculation).

\begin{tabular}{|c|c|c|c|c|c|c|}
\hline \multirow{2}{*}{ No Block } & \multicolumn{3}{|c|}{ Before Identification } & \multicolumn{3}{|c|}{ After Identification } \\
\hline & $\alpha$ & $\lambda_{a q}, \mathrm{~m}^{3} / \mathrm{atm} /$ Month & $J$ & $\alpha$ & $\lambda_{a q}, \mathrm{~m}^{3} / \mathrm{atm} /$ Month & $J$ \\
\hline 1 & 0.80 & 100 & 0.18 & 0.60 & 0 & 0.04 \\
\hline 2 & 0.80 & 100 & 0.07 & 0.81 & 100 & 0.07 \\
\hline 3 & 0.80 & 100 & 0.13 & 0.99 & 298 & 0.04 \\
\hline 4 & 0.80 & 100 & 0.07 & 0.65 & 390 & 0.04 \\
\hline 5 & 0.80 & 100 & 0.16 & 1.00 & 235 & 0.14 \\
\hline 6 & 0.80 & 100 & 0.38 & 0.60 & 0 & 0.30 \\
\hline
\end{tabular}

Table 1. Calculated parameters. 
As a result of minimizing function (14), taking into account Equation (3) in the first stage application of the algorithm described above-for each of the blocks- the identified values of the bottom water activity coefficient $\lambda_{a q}$ and the effective injection coefficient $\alpha$ were obtained. In the second stage of identification, the coefficient $\lambda_{i, j}$, was determined, characterizing the intensity of flows between the blocks (Figure 6). The values of the objective function at various stages of identification are presented in Figures 9 and 10, which show how the values of the average reservoir pressures changed after the stage-bystage material balance calculation.


Figure 9. Results of step-by-step identification. The values of the minimized function for each block are shown: 1-without identification (J at the initial values of the control parameters); 2 -after the first stage of identification; 3-after the second stage of identification.

Comparing the values of the objective function separately for each block, obtained in the first stage of the algorithm, it is evident that the greatest deviation of the calculated pressure from the actual one is for the sixth block (Figure 9). However, after the second stage of identification, the value of the objective function for the sixth block decreased significantly. In the remaining areas, the changes in the objective function are not so noticeable, the difference in its values after the first and second stages of the algorithm is minimal.

Thus, the following parameters that characterize the efficiency of reservoir development have been determined: the coefficient of efficiency of water injection into the reservoir, the aquifer activity, reservoir pressure and transmissibility between the blocks. Using these parameters, fluid flow between individual blocks, between blocks and the aquifer can be calculated. Information on fluid flow between blocks can be used for sector hydrodynamic modeling when boundary conditions are corrected. 

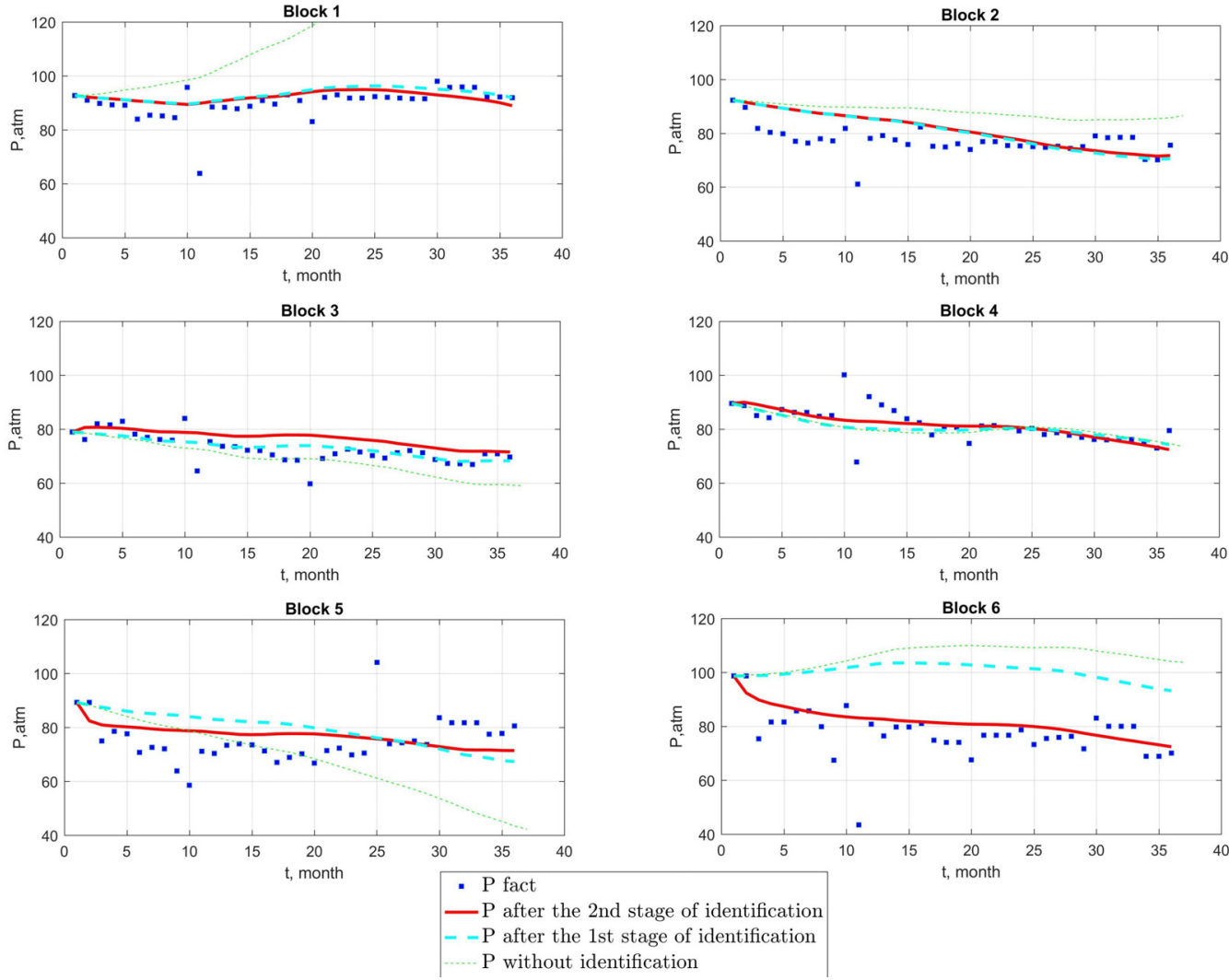

Figure 10. Comparison of actual and calculated reservoir pressures at each stage of identification for all oil field blocks.

\section{Discussion and Conclusions}

This paper presents a description of simplified material balance models for a homogeneous fluid, and also proposes an approach to solving the problem of identifying these models. Material balance models are considered at various levels of spatial detail: for the field as a whole, for hydrodynamically connected blocks, for hydrodynamically connected wells. Each of these models has its own area of application, and, accordingly, its own range of tasks, which can be solved using these models. Thus, the material balance model for the field is used to assess the reservoir energy state; if there are blocks on the object and they are hydrodynamically connected to each other, then at the initial stage it is possible to calculate the fluid flows between blocks, and, using the calculated flows as boundary conditions, isolate this block for more detailed study/modeling. Further, the material balance model for wells makes it possible to estimate the average interwell transmissibility, and, for instance, after the history matching procedure, will be able to predict the production rates if there are known injection flow rates and reservoir pressure dynamics. Using the material balance model for a system of hydrodynamically connected wells, one of the most difficult and urgent problems facing hydrodynamic modeling is being solved, namely: the problem of the waterflooding optimization. This problem, as a rule, is solved within the framework of full physics modeling, however, compared to FFM, material balance models require significantly less input data, calculations are performed much faster and require significantly less computing resources.

When solving the inverse ill-posed problem, even for simplified models, the solutions obtained are non-uniqueness. In the work, using an isolated block as an example, it was demonstrated that the problem of multicollinearity of the coefficients that determines the intensity of bottom water inflow and the effective injection arises, i.e., it is impossible to determine a unique solution from the set obtained. When using more complex models, the number of matched parameters increases quadratically relative to the number of equations in the system. To solve this ill-conditioned identification problem, it is necessary to involve 
additional regularizing information about the reservoir or properties of the evaluated solution. One of the methods implemented in the article is statistical processing of the obtained solutions for the stability of the appearance of connections between wells and obtaining criteria for determining the connectivity matrix, and, accordingly, the selection of models. The hierarchical approach assumes that solutions obtained in models from the previous level of the hierarchy can be used as regularizing information for a more complex model. This approach was implemented using the example of a step-by-step calculation of the material balance for blocks, where, firstly, the parameters that determine the effective injection and the intensity of bottom water inflow in isolated blocks are determined. Secondly, these parameters are "frozen" and are not identified when calculating the connections between blocks. This approach made it possible to regularize the ill-conditioned inverse problem, significantly reduce the number of simultaneously identifiable parameters, and, ultimately, evaluate a unique solution.

Author Contributions: Conceptualization, E.N.M. and S.P.R.; methodology, E.N.M. and S.P.R.; software, E.N.M.; validation, S.P.R. and N.G.M.; formal analysis, E.N.M., S.P.R. and N.G.M.; investigation, E.N.M. and S.P.R.; writing - original draft preparation, E.N.M. and N.G.M. All authors have read and agreed to the published version of the manuscript.

Funding: Computational experiments and analysis of the results were carried out within the framework of a grant from RFBR and Tyumen Region, project number 20-41-720004. Formulation of the problem was carried out within the state assignment of Ministry of Science and Higher Education of the Russian Federation (project No. 121030500156-6).

Institutional Review Board Statement: Not applicable.

Informed Consent Statement: Not applicable.

Data Availability Statement: The data presented in this study are available on request from the corresponding author.

Conflicts of Interest: The authors declare no conflict of interest.

\section{Nomenclature}

$B \quad$ conditional width of the wall through which the liquid is filtered $(\mathrm{m})$

$H \quad$ arithmetic mean of the effective reservoir thickness (m)

K absolute permeability at the boundary between the wells $\left(\mathrm{m}^{2}\right)$

$L \quad$ determines distance between wells (m)

m porosity

$N \quad$ number of interwell connections (wells, blocks)

$P \quad$ pressure $(\mathrm{Pa})$

$Q \quad$ volumetric flow $\left(\mathrm{m}^{3} \mathrm{~s}^{-1}\right)$

$S \quad$ saturation

$T \quad$ transmissibility $\left(\mathrm{m}^{3} \mathrm{~Pa}^{-1} \mathrm{~s}^{-1}\right)$

$V \quad$ pore volume $\left(\mathrm{m}^{3}\right)$

Greek Symbols

$\alpha \quad$ effective injection

$\beta \quad$ effective compressibility $\left(\mathrm{Pa}^{-1}\right)$

$\kappa \quad$ parameter of the model complexity or the number of identifiable parameters

$\lambda \quad$ coefficient, determining the intensity of inflow $\left(\mathrm{m}^{3} \mathrm{~Pa}^{-1} \mathrm{~s}^{-1}\right)$

$\mu \quad$ viscosity (Pa s) 


\section{References}

1. Zhang, H.L.; Liu, C.X.; Zhao, M.Z.; Sun, Y. Economics, fundamentals, technology, finance, speculation and geopolitics of crude oil prices: An econometric analysis and forecast based on data from 1990 to 2017. Pet. Sci. 2018, 15, 432-450. [CrossRef]

2. Musakaev, N.G.; Borodin, S.L. Mathematical model of the two-phase flow in a vertical well with an electric centrifugal pump located in the permafrost region. Heat Mass Transf. 2016, 52, 981-991. [CrossRef]

3. Wang, Y.; Ren, S.; Zhang, L. Mechanistic simulation study of air injection assisted cyclic steam stimulation through horizontal wells for ultra heavy oil reservoirs. J. Pet. Sci. Eng. 2019, 172, 209-216. [CrossRef]

4. Shagapov, V.S.; Musakaev, N.G.; Khabeev, N.S.; Bailey, S.S. Mathematical modelling of two-phase flow in a vertical well considering paraffin deposits and external heat exchange. Int. J. Heat Mass Transf. 2004, 47, 843-851. [CrossRef]

5. Turkyilmazoglu, M. Effective computation of exact and analytic approximate solutions to singular nonlinear equations of Lane-Emden-Fowler type. Appl. Math. Model. 2013, 37, 7539-7548. [CrossRef]

6. Aziz, K.; Settari, A. Petroleum Reservoir Simulation; Springer: Amsterdam, The Netherlands, 1979.

7. Turkyilmazoglu, M. Parametrized Adomian Decomposition Method with Optimum Convergence. ACM Trans. Modeling Comput. Simul. 2017, 27, 21. [CrossRef]

8. Williams, G.J.J.; Mansfield, M.; MacDonald, D.G. Top-Down Reservoir Modelling. In Proceedings of the SPE Annual Technical Conference and Exhibition, Houston, TX, USA, 26-29 September 2004.

9. Gavris, A.S.; Kosyakov, V.P.; Botalov, A.Y.; Pichugin, O.N.; Rodionov, S.P.; Sokolyuk, L.N.; Shirshov, Y.V. The concept of effective design of hydrocarbon fields development. Software solutions. Oilfield Eng. 2015, 11, 75-85. (In Russian)

10. Holanda, R.W.; Gildin, E.; Jensen, J.L.; Lake, L.W.; Kabir, S. A State-of-the-Art Literature Review on Capacitance Resistance Models for Reservoir Characterization and Performance Forecasting. Energies 2018, 11, 3368. [CrossRef]

11. Moreno, G.A.; Lake, L.W. On the uncertainty of interwell connectivity estimations from the capacitance-resistance model. Pet. Sci. 2014, 11, 265-271. [CrossRef]

12. Artun, E. Characterizing interwell connectivity in waterflooded reservoirs using data-driven and reduced-physics models: A comparative study. Neural Comput. Appl. 2017, 28, 1729-1743. [CrossRef]

13. Klie, H. Physics-Based and Data-Driven Surrogates for Production Forecasting. In Proceedings of the SPE Reservoir Simulation Symposium, Houston, TX, USA, 23-25 February 2015.

14. Rafiee, M.M. Model Selection and Uniqueness Analysis for Reservoir History Matching. Ph.D. Thesis, Technische Universität Bergakademie Freiberg, Freiberg, Germany, 2010.

15. Mohaghegh, S.D.; Gaskari, R.; Maysami, M.; Khazaeni, Y. Data-Driven Reservoir Management of a Giant Mature Oilfield in the Middle East. In Proceedings of the SPE Annual Technical Conference and Exhibition, Amsterdam, The Netherlands, 27-29 October 2014. [CrossRef]

16. Naudomsup, N.; Lake, L.W. Extension of Capacitance-Resistance Model to Tracer Flow for Determining Reservoir Properties. In Proceedings of the SPE Annual Technical Conference and Exhibition, San Antonio, TX, USA, 9-11 October 2017. [CrossRef]

17. Gildin, E.; King, M. Robust Reduced Complexity Modeling $\left(\mathrm{R}^{2} \mathrm{CM}\right)$ in Reservoir Engineering. In Proceedings of the Foundation CMG Summit, Calgary, AB, Canada, 24-25 June 2013.

18. Musakaev, E.N.; Rodionov, S.P.; Kosyakov, V.P. Structure and parameter identification technique for modeling problems of two-phase filtration in porous media. AIP Conf. Proc. 2018, 1939, 020053. [CrossRef]

19. Zhao, H.; Kang, Z.; Zhang, X.; Sun, H.; Cao, L.; Reynolds, A. INSIM: A Data-Driven Model for History Matching and Prediction for Waterflooding Monitoring and Management with a Field Application. In Proceedings of the SPE Reservoir Simulation Symposium, Houston, TX, USA, 23-25 February 2015.

20. Guo, Z.; Reynolds, A.C.; Zhao, H. A Physics-Based Data-Driven Model for History-Matching, Prediction and Characterization of Waterflooding Performance. In Proceedings of the SPE Reservoir Simulation Conference, Montgomery, TX, USA, 20-22 February 2017.

21. Lerlertpakdee, P.; Jafarpour, B.; Gildin, E. Efficient Production Optimization with Flow-Network Models. SPE J. 2014, 19, 1083-1095 [CrossRef]

22. Kaviani, D.; Valkó, P.P.; Jensen, J.L. Application of the Multiwell Productivity Index-Based Method to Evaluate Interwell Connectivity. In Proceedings of the SPE Improved Oil Recovery Symposium, Tulsa, OK, USA, 24-28 April 2010.

23. Ahmed, T. Reservoir Engineering Handbook; Gulf Professional Publishing: Cambridge, MA, USA, 2018.

24. Mirzajanzade, A.K.; Khasanov, M.M.; Bakhtizin, R.N. Modeling of Oil and Gas Production Processes. Nonlinearity, Disequilibrium, Uncertainty; Institute of Computer Research: Moscow-Izhevsk, Russia, 2004. (In Russian)

25. Kosyakov, V.P.; Musakaev, E.N.; Shirshov, Y.V. Computational technology for calculating the material balance in an oil field. Oilfield Eng. 2015, 11, 30-35. (In Russian)

26. Musakaev, E.N.; Rodionov, S.P.; Legostaev, D.Y.; Kosyakov, V.P. Parameter identification for sector filtration model of an oil reservoir with complex structure. AIP Conf. Proc. 2019, 2125, 030113. [CrossRef]

27. Rodionov, S.P.; Musakaev, E.N.; Kosyakov, V.P. New Technology of Interwell Connectivity Identification in Oil Reservoir Modelling; EAGE: Gelendzhik, Russia, 2018.

28. Musakaev, E.N.; Rodionov, S.P.; Kosyakov, V.P. Problem of structure and parameter identification at simulation of two-phase filtration in porous media. Proc. Cybern. 2018, 1, 39-49. (In Russian) 